\title{
Role of Artificial Intelligence in Indian Agriculture: A Review
}

Vinod Chandra S.S.

10.18805/ag.R-2296

\begin{abstract}
The next generation is very keen on agriculture and is looking forward to high yield productivity by using the latest technologies. Artificial intelligence tops the list as it creates an eco-system that is favourable to all stakeholders involved. Technology should produce a framework that helps the farmers in every aspect of cultivation, starting from land preparation to selecting seeds to fertilize. The use of technology can help them to identify the apt plantations for each season. Market analysis and user demands can also be foreseen by the farmers, which helps them get a better yield. Artificial intelligence-based farming creates high productivity and better yield, which increases the profit of individual farmers. An end-end market where the customer gets quality products directly from the producers increases the quality and reduces the price hike. Technology-driven farming will pull the next generation to take up agricultural jobs shortly. This method will help farmers to understand the demand of the market through prediction forecasting. Artificial intelligence and its allied fields are tuned to 'agro computer' in tomorrow's world.
\end{abstract}

Key words: Agriculture, Agro-computer, Agro-market, Artificial intelligence, Sos framework.

Agriculture is the backbone of our country's economy and nearly $70-75 \%$ depend directly or indirectly on it. Even in a country like ours, it is hard to believe that methods and markets of agriculture are way low the basic standards. There are no proper guidelines available for farming. No proper market study and not even scientific farming methods are reaching the farmers. Markets and technologies are never with the producers and they are not even aware of new farming methods. Israel is a very good example where advanced farming methods are employed for high yielding. Here in India, not even our agricultural research institutes are developing technologies that help agriculture.

Let's see how artificial intelligence and expert systems can contribute to the country's agricultural development. When talking about intelligence, human intelligence is one of the most complex systems. The two aspects of intelligence are learning and dealing with things and the ability to rationalize thought. Artificial intelligence is a computer science branch with many applications in different fields (Vinod and Anand, 2020). It learns how to program computers to behave intelligently. It has to do with a job similar to using computers to understand human intelligence.

What are the use and use of artificial intelligence for the common person? Of course, in today's world, the use of artificial intelligence is useful. Although difficult for the common person to understand, artificial intelligence profoundly affects everyone in society and plays a vital role in progressive modern life. High tech agriculture tools, smart transporting and consistent field analytics to farmers and insurers will help agriculture bloom. In digital framing, high yield productivity is expected. It applies precision location methods and decision quality agronomic information to brighten, predict and affect the range of cultivation issues across the farm.

Deploying science and technology in agriculture is essential to eradicate severe poverty and famine. As part of a study, Microsoft Corporation has been operating in Andhra
Department of Computer Science, University of Kerala, Thiruvananthapuram-695 034, Kerala, India.

Corresponding Author: Vinod Chandra S.S., Department of Computer Science, University of Kerala, Thiruvananthapuram-695 034, Kerala, India. Email: vinod@keralauniversity.ac.in

How to cite this article: Vinod Chandra, S.S. (2022). Role of Artificial Intelligence in Indian Agriculture: A Review. Agricultural Reviews. DOI: 10.18805/ag.R-2296.

Submitted: 04-06-2021 Accepted: 24-12-2021 Online: 20-01-2022

Pradesh, India, since 2018, providing services and solutions to 175 farmers (Gurumurthy and Bharthur, 2019). In collaboration with International Crops Research Institute for the SemiArid Tropics (ICRISAT), Microsoft developed an artificial intelligence sowing App that uses machine learning and business intelligence from the Microsoft Cortana Intelligence Suite. It uses powerful cloud-based predictive analytics to empower farmers with crucial information and insights to reduce crop failure, increase yield, reduce stress and make better income (Diksha, 2020). It involves land preparation, sowing of seeds, climate change, fertilizer addition, software application, organic fertilizers and other nutrients for the crop. On average, the yield per hectare has increased by $30 \%$ compared to the previous harvest.

Predictive analytics will help the framers for timely crop sowing, make predictions on the right time to plant, apply fertilizers, harvest, etc., based on climate data, historical conditions, market conditions, personal information, etc. With full knowledge and consent, digital technologies can have conquered the agricultural sector. But right now, we are in a phase where artificial intelligence is entering the Indian agricultural sector without much groundwork. This will badly affect our farmers and markets. Mainly our agricultural community, primarily farmers and those who 
depend on them. The second impact will be on the agricultural market and those who depend on it. Access to the market through unreliable market connectivity, fluctuations in commodity prices, low income and high debt make agriculture a high-risk factor. In the nineteenth century, during the Industrial Revolution, machines were deployed to reduce human resources. Now in this twenty-first century, demands are for modern methods and technological updations used in agriculture.

The primary experimental study was conducted during 2018-2020 along with Kerala Agricultural University and the Department of Computer Science, University of Kerala. The review materials were selected by the help of agricultural scientists of the Kerala Agricultural University.

\section{Technologies in agriculture}

Artificial intelligence and loT based remote access micro-grid power farming has slowly started in India. Indian government organizations like C-DAC is providing facilities to farmers aimed at promoting agriculture. New technologies need to be implemented as the demand for agricultural products has increased over time. Crop productivity has increased over time as a result of advancements in information technology, which resulted in producing field yield seeds. The use of computers played a significant role in heavy crop production in the twentieth century. There is no doubt that artificial intelligence will play a role in the next decades. It is a fact that artificial intelligence offers ways to facilitate an increase in farmers' incomes, a rise in agricultural productivity and even waste reduction. Artificial intelligence plays a significant role in all these areas, thus preventing "agriculture as a service" from being a fragile segment. The uses of cognition are spread over eight main areas. This will benefit the Indian agricultural sector. They need to come up with technologies, especially artificial intelligence.

1. Internet of Things (loT) led growth.

2. Image-based insight work.

3. Prepare suitable mixtures for agricultural products.

4. Strengthen crop health monitoring

5. Enable farmers to use irrigation and its maximum use.

6. Implement self-sustaining technology projects in agriculture.

7. Direct value-added products and manufacturing and enterprise marketing in the right direction.

8. Understand the right direction of the market.

The demand for data-driven framing and predictive analytics in agriculture is a guide after the post-COVID 19 worlds. The framers and agro-business owners are actively taking up precision farming methods supported by artificial intelligence and machine learning frameworks. These frameworks support farmers in crop and livestock management by removing the element of guesswork, forecasting yield, managing a supply chain network and assessing risks (Saxena et al. , 2020). Blockchain technology gives a transparent supply chain in various agriculture fields, starting from seed identification to harvest. It will provide popularity among the framers and systems regarding technical aspects, education, policies and regulatory frameworks.

\section{Challenges in adopting technologies in agriculture}

The Niti Aayog, Government of India, recently published a discussion paper (Tanay, 2019). It envisions solutions using artificial intelligence for key areas, including agriculture, industrialization, etc. In agriculture, technologically advanced machinery and built-in intelligence give farmers knowledge about soil quality, when to sow, spray herbicides, where pests occur, etc. If we built an intelligent system, then it can advise farmers on various best practices, India thus can see a new agricultural revolution. However, there is a strong challenge for that futuristic situation. The entire supply chain uses capacity expansion and cost reduction factors, which can backstab the Indian farming population. Even though built-in intelligence-based technologies have unique advantages, there are several challenges in adopting them in the agricultural sector (Lal Mohan et al., 2019):

1. Reliability of system and technology.

2. Security and acceptance of information.

3. Data privacy and storage and its use.

4. Social acceptance and recognition.

5 . Live release, accessibility and use of reliable information.

6. Cost-effectiveness.

7. Ease of use and training.

8. Unethical stakeholders.

We must realize the realities of Indian agriculture and its market. Farmers are dependent on various external factors for agriculture and harvest and many a time, they are not guaranteed, which makes farming a high-risk activity. Even the market plays a vital role; since the products are mostly perishable, they have to accept whatever the market offers. Lack of quality storage facilities also is a reason why the farmers are getting exploited by the market. Certain agro-products like milk, egg, meat and vegetables will be damaged after a limited time. If artificial intelligencebased techniques were deployed, it would have given optimum solutions in cold storage, transportation and demand for these products. For example, every place has its festivals. The market need of that time is different and if it can be predicted, then the demand and supply could be foreseen and a new market opportunity could be fetched.

Application of technology farmers in India have a very small landholding and they are unable to sustain the cost of buying the seeds and other essentials. Here, artificial intelligence for predicting the weather at a local level guides farmers to use sustainable techniques (Tanha et al, 2020). This helps framers manage pests through ecology, robots for harvesting in a multi-crop farm, demand prediction available stocks, export, local needs, etc.

The importance of agriculture demands in the world increases because of impact issues after COVID 19. Technological advancements in agriculture farming and its marketing lead to International economic growth and the prevailing lockdown situation worldwide. It further resulted in food supply chain disruptions using predictive techniques. 


\section{Artificial intelligence models for agriculture}

Globally, built-in technologies are emerging to improve the efficiency of agriculture, seed quality, soil monitoring, climate forecasting, forecast agricultural analysis, markets and distribution networks. We can use cloud computing infrastructure, data eco-systems, the Internet of Things (IoT) and machine learning to make more productive farming. We can focus on the development of the digital agricultural sector. For this, focus on efficient farming, efficient irrigation, smart chemical spraying, effective harvesting and pest control. Artificial intelligence-based automated irrigation dramatically increases the efficiency of the current agriculture supply chain. The percentage of freshwater resource requirements increased with the increase in food demand. Hence, artificial intelligence plays an importance in smart irrigation, water level, soil temperature, nutrient content and weather forecasting (Tanha et al, 2020). Models can be created to achieve such demands. Machine learning and soft computing techniques, a subfield of artificial intelligence, can be adapted for model building. Use of plant growth images/videos, we can make intelligence models using the above techniques. Built-in intelligence is widely used worldwide for drone cameras, satellite imagery, data processing, systems for monitoring and managing various agricultural activities, disease and pest detection, weather forecasts and fertilizer application times. A sub-branch of artificial intelligence can predict the proper use of chemical sprays, harvest time and product life through machine learning. For example, the harvest time and weight of bananas grown on the same soil on the same day are different. This can happen even if the fertilizer is applied evenly. Such problems can be technically examined and resolved. The complexity increases when multiple such farming problems are combined. These problems can be solved by building and using specialized built-in intelligence models with the necessary learning. In-depth study methods using agricultural data, crop diagrams, growth rates and disease data provide useful forecasting assumptions on market and harvest (Hatim et al., 2019).

Today banking and financial institutions give artificial intelligence-based lending solutions to the farmers. The payback capacity can identify before sectioning the amount using the data analytics by machine learning. Similarly, insurance companies are using machine learning frameworks to augment the processes related to the current crop insurance schemes, preventing the claimed delay and reducing the timeline in claim settlements.

Robots and intelligent machines are useful for farming and harvesting, leading to huge savings by reducing need for agricultural laborers. Identify the right seeds' availability for the next seed germination and flowering machine learning framework for Indian farmers. For example, many seed banks in India need to coordinate with a machine learning framework for distribution to the framers. The seed suggestions are based on that farming place's soil structure, availability, market and weather conditions.

\section{Artificial intelligence-based framework}

The Government's Economic Survey 2020 identified the data-based facility as a critical characteristic (Arka, 2018). It underscored the desire to build things based on people's data. Agriculture and the agricultural market focused on it. This is where the open potential for the study of our artificial intelligence in agriculture lies. For making a framework, acceptable working practices are required. Through our research, agriculture and farming methods will benefit the country as a development model under artificial intelligence supervision. This is being implemented in other developed countries.

A model framework (systems of sedentary behaviours or SoS) is shown in Fig 1. The Indian monsoon rainfall is predicted using artificial intelligence techniques such as artificial neural networks. Indian meteorological department has developed rainfall and climate change forecasting methods. Including these technologies into the SoS framework, the framers get a maximum yield and agricultural profit. This can be done in many ways. In this way, a sense of direction can be given to the next generation. Natural language processing (NLP), robotics, machine learning automatic reasoning, knowledge representation, expert systems, computer vision, speech recognition, automated data analytics and cloud computing, statistical computing and deep learning are some of the key subfields of artificial intelligence that can be used to solve complex problems in agriculture (Konstantinos et. al., 2018). Many of these are used by our farmers through technological software. For example, a stand-alone Q\&A system in Indian languages for farmers. The deep learning-based agriculture mapping from satellite images gives an intelligent forecasting system to the farmers and agro-business. It is built on top of an image data stream to help recognize soil science, crop yield, pest management, harvesting, etc.

Precision agriculture focused on improving the overall harvest quality and accuracy through artificial intelligence methods. The detection of target weeds can be by artificial intelligence sensors and then routed to herbicide region selection. This is by automation mechanism to the agriculture farming. The storage of seeds for the next season is one of the challenging tasks. Using data analytics by machine learning techniques provide a clear insight to the distributors. Similarly, the storage and distribution of grains to the market and users' need an intelligent forecasting technique. Forecast the price of agro-products in the present and futures markets are today's requirements. Also, strengthening the health of crops is challenging. An artificial intelligence-based framework will help those farmers by suggestions. Crop diseases are a severe problem that leads to low yield, productivity and unrespecting causes to the local farmers (Selvaraj et.al., 2019). Better identification of crop disease in advance using machine learning techniques offers remedial solutions to high productivity farmers. 


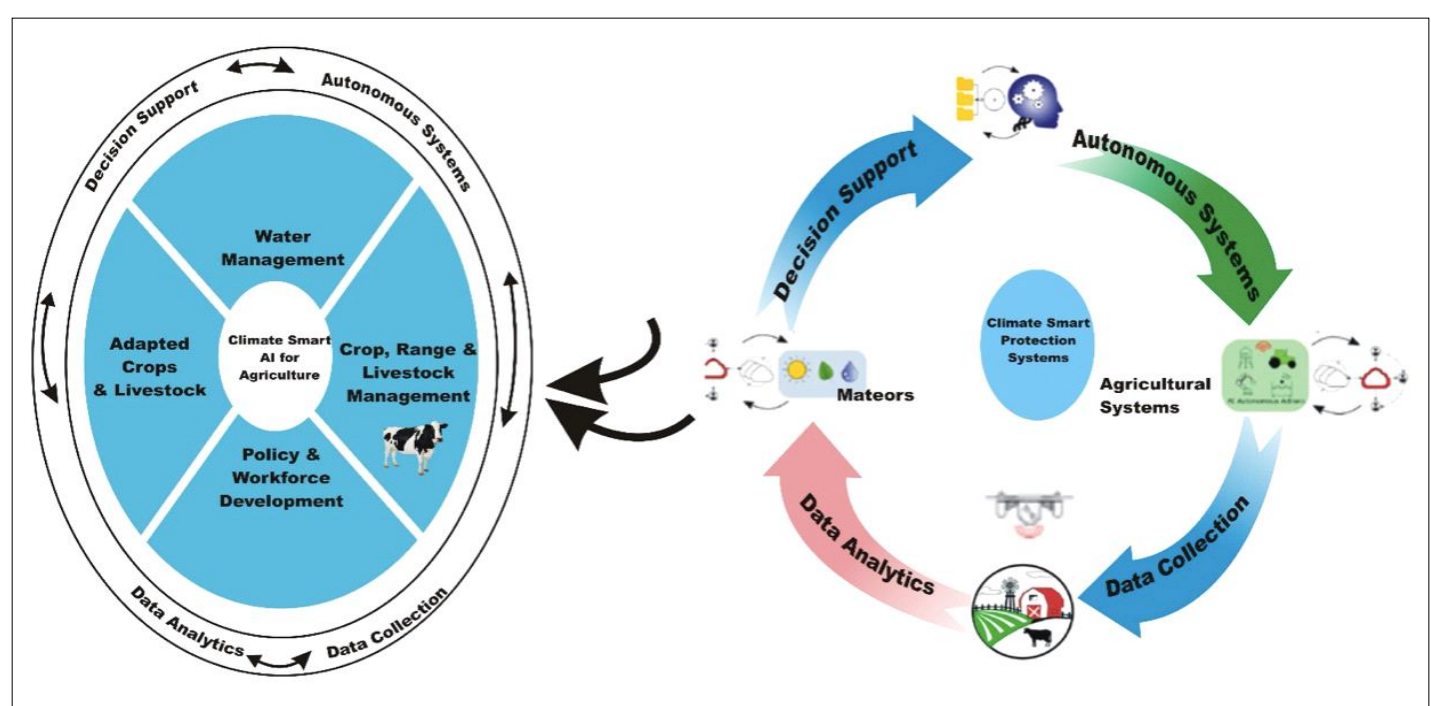

Fig 1: Artificial intelligence-based SoS.

\section{Challenge of Artificial Intelligence Research}

One of the major challenges is how artificial intelligence research is carried out in our country. Current research and development initiatives are concentrated in top Indian institutions such as the Indian Institute of Technology (I.I.T), National Institute of Technology (N.I.T), etc. Here a community of 50 to 75 people works in this domain, and with this small workforce, they cannot pull off sufficient advancements. Thus, India lags far behind in bringing high-quality outputs in smart and intelligent computation. Space, lack of resource and administrative barriers, approach to research, poor computing facilities, lack of interpreted quality data have been cited as major pain affecting India's artificial intelligence research. The increase in Digital India's budget to US \$ 477 million in 2018 to expand artificial intelligence research was a welcoming first step. But even with these funds, addressing institutional weaknesses continue to be a major challenge. It is also not clear how public agricultural universities and research institutes will benefit from such allocation. Artificial intelligence applications in the Indian agricultural sector need to grow, and the technologies need to be brought down to the root level. All stakeholders should be benefitted. Technologies should never remain in laboratories, and it should be handed down and tested in reality. Recommendation systems will benefit consumers and farmers in identifying the entire supply chain, which will quickly benefit both parties. If we adopt artificial intelligencedriven methodologies at different farming stages, we can witness an era of flourishing agricultural resources. This can only be achieved by advancing multiple research methods simultaneously.

\section{CONCLUSION}

Today's world is moving to next-generation agriculture by using artificial intelligence framework, which can be termed as smart agriculture system. For better yield in agriculture, it is recommended,

1. Increase support for research and development activities in the area of artificial intelligence in agriculture.

2. Implementing expert guidance strategies, large scale research and application units.

3. Effective evaluation of equipment using artificial intelligence and related methodologies.

4. Improve interaction with all stakeholders at multiple levels of the supply chain.

5. Provision for agro educational research efforts.

6. A holistic system approach enables scalability and rapid response to changes in water, climate and employment availability, i.e., adopting an SoS framework.

7. Development of secure, adaptive and cohesive systems.

8. Understand the innovative and intelligent processes that take place at various agricultural universities and their impact on agriculture.

9. Understand the term "agricultural computer" on an experimental basis. That is, see the interaction of artificial intelligence and sub-sectors in the agricultural domain.

10. Adopt an integrated, multi-dimensional research approach in agriculture.

\section{REFERENCES}

Arka Bagchi (2018). Artificial Intelligence in Agriculture, Mindtree white paper, (2018).

Konstantinos Liakos, Patrizia Busato, Dimitrios Moshou,Simon Pearson and Dionysis Bochtis (2018). Machine Learn ing in Agriculture: A Review, Sensors in Agriculture, 18, 2674; DOI: doi.org/10.3390/ s18082674.

Diksha Manaware (2020). Artificial Intelligence: A New Way to Im prove Indian Agriculture. International Journal of Current Microbiology and Applied Sciences, 9(3): 1095-1102. DOI:10.20546/ ijcmas.2020.903.128.

Gurumurthy, Anita and Bharthur, Deepti (2019). Taking Stock of Artificial Intelligence in Indian Agriculture. Friedrich-EbertStiftung, DOI:10.13140/RG.2.2.13914.36801. 
Hatim, G., Lara, P., Jennifer, R. and Son, C. (2019). Climate adaptive smart systems for future agricultural and rangeland production. White Paper, (2019).

Lal, M.B, Ramasubramanian, V., Arora, A., Marwaha, S. and Parsad, R. (2019). Era of Artificial Intelligence: Prospects for Indian Agriculture. Krishi. 69(3): 10-13, DOI: krishi.icar.gov.in /jspui/handle/123456789/19207.

Selvaraj, M., Alejandro, V., Henry, R., Nancy, S., Sivalingam, E., Walter, O. and Guy, Blomme, (2019). Al-powered banana diseases and pest detection. Plant Methods. 15: 92. DOI: doi.org/10.1186/s13007-019-0475-z.

Saxena, A., Suna, T. and Saha, D. (2020). Application of Artificial
Intelligence in Indian Agriculture, RCA Alumni Association, India.

Tanay, M. (2019). Role of Digital and Al Technologies in Indian Agriculture: Potential and way forward, NITI Aayog, September (2019).

Tanha, T., Dhara, S., Patel, N., Yagnik, H. and Shahd, M. (2020). Implementation of artificial intelligence in agriculture for optimization of irrigation and application of pesticides and herbicides. Artificial Intelligence in Agriculture. 4: 58-73. DOI: 10.1016/j.aiia.2020.04.002.

Vinod Chandra, S.S., Anand, H.S. (2020). Artificial Intelligence: Principles and Applications, Printice Hall India, pp.428. 